\title{
Early phase II study of robot-assisted distal gastrectomy with nodal dissection for clinical stage IA gastric cancer
}

\author{
Masanori Tokunaga $\cdot$ Norihiko Sugisawa $\cdot$ \\ Junya Kondo • Yutaka Tanizawa $\cdot$ Etsuro Bando • \\ Taiichi Kawamura $\cdot$ Masanori Terashima
}

Received: 13 March 2013/Accepted: 4 August 2013/Published online: 5 September 2013

(C) The International Gastric Cancer Association and The Japanese Gastric Cancer Association 2013

\begin{abstract}
Background Robot-assisted distal gastrectomy (RADG) is increasingly performed in Japan and Korea and is thought to have many advantages over laparoscopic gastrectomy. However, a prospective study investigating the safety of RADG has never been reported. The present study evaluated the safety of RADG with nodal dissection for clinical stage IA gastric cancer.

Methods This single-center, prospective phase II study included patients with clinical stage IA gastric cancer located within the lower two-thirds of the stomach. The primary endpoint was the incidence of postoperative intraabdominal infectious complications including anastomotic leakage, pancreas-related infection, and intraabdominal abscess. The secondary endpoints included all inhospital adverse events, RADG completion rate, and survival outcome.

Results From May 2012 to November 2012, 18 eligible patients were enrolled for this study. The incidence of intraabdominal infectious complication was $0 \%(90 \% \mathrm{CI}$, $0-12.0 \%)$. The overall incidence of in-hospital adverse events was $22.2 \%$ (90\% CI, 8.0-43.9\%). No patient required conversion to laparoscopic or open gastrectomy; thus, the RADG completion rate was $100 \%$.

Conclusions This early phase II study suggested that RADG might be a safe and feasible procedure for stage IA gastric cancer, providing experienced surgeons perform the
\end{abstract}

M. Tokunaga $\cdot$ N. Sugisawa $\cdot$ J. Kondo $\cdot$ Y. Tanizawa

E. Bando $\cdot$ T. Kawamura $\cdot$ M. Terashima $(\varangle)$

Division of Gastric Surgery, Shizuoka Cancer Center,

1007 Shimonagakubo, Nagaizumi-cho, Sunto-gun,

Shizuoka 411-8777, Japan

e-mail: m.terashima@scchr.jp surgery. This conclusion should be clarified in subsequent late phase II studies with a larger sample size.

Keywords da Vinci · Gastric cancer · Gastrectomy · Clinical trial $\cdot$ Safety

\section{Introduction}

Laparoscopy-assisted distal gastrectomy (LADG) is performed increasingly often, particularly in East Asian countries where the incidence of early gastric cancer is higher than in Western countries. The safety of LADG was clarified by prospective studies [1, 2], and survival outcome of LADG compared with open gastrectomy was under investigation in two large, nationwide, randomized controlled trials in Japan and Korea [1, 3]. However, current laparoscopic procedures have several drawbacks, including a limitation in range of forceps movement and the two-dimensional surgical view available to the operating surgeons.

Robot-assisted distal gastrectomy (RADG) may enable us to overcome these drawbacks. Using the da Vinci Surgical System (Intuitive Surgical, Sunnyvale, CA, USA), surgeons were able to attain a three-dimensional surgical view, instrument flexibility, tremor suppression, and improved ergonomics, although RADG still has disadvantages such as high cost and lack of tactile sensation [4-8]. In addition, a shorter learning curve has been reported for robotic surgery compared to laparoscopic surgery [9-11].

Reported studies rate RADG as a feasible procedure, although most such studies involved a retrospective or prospective study cohort $[4,5,8-10,12-22]$. So far, no prospective clinical trials have focused on the feasibility of RADG, a step that is necessary before RADG could be 
explored further with greater number of patients. To this end, the current prospective study evaluated the safety of RADG with nodal dissection for clinical stage IA gastric cancer.

\section{Methods}

The present study was designed as a single-center, prospective phase II trial. The institutional review board of Shizuoka Cancer Center approved the study protocol, which had the following inclusion criteria: histologically confirmed adenocarcinoma of the stomach, clinical stage IA early gastric cancer according to the International Union Against Cancer classification system (UICC) [23], no indication for endoscopic submucosal dissection (ESD), a tumor located in the lower two-thirds of the stomach, no involvement of the duodenum, patient age of 20-80 years, an Eastern Cooperative Oncology Group (ECOG) performance status (PS) of 0 or 1, a body mass index (BMI) less than $30 \mathrm{~kg} / \mathrm{m}^{2}$, no prior upper abdominal surgery or intestinal resection other than appendectomy, no prior chemotherapy or radiotherapy for any malignancy, adequate organ function, and written informed consent. The study was registered with clinical trials.gov (clinical trials.gov identifier: NCT 1504997).

In this study period, medical cost for hospital admission, including surgical fee, was funded by the Shizuoka Cancer Center because the national insurance system in Japan did not reimburse patients for RADG.

Surgical procedure

All RADG operations were performed using the da Vinci Surgical System with four robotic arms; a central arm for a dual-channel endoscope, and the other three for a Cadiere forceps, fenestrated bipolar forceps, and bipolar Maryland forceps or monopolar electrocautery, respectively. One assistant port was placed in the right umbilical level. The surgical procedures were similar to that used in LADG, with a standardized surgical field to achieve omentum preservation, D1+ lymph node dissection according to the Japanese gastric cancer treatment guidelines [24-27], and vagal nerve preservation [28, 29]. Removal of resected specimens and reconstruction were performed by a 4- to 5 -cm upper midline incision. In the case of distal gastrectomy, a Billroth I reconstruction with circular stapler was selected in general. In the case of pylorus-preserving gastrectomy, reconstruction was performed by hand-sewn sutures.

In this study, the operations were separated into three parts. The docking time was defined as the time from skin incision to completion of docking. The console time was the time that the da Vinci system was used by the surgeon at the console. The anastomosis time was the time spent from the creation of the mini-laparotomy to the completion of the surgery.

\section{Training for RADG}

A team of two gastric surgeons who were board certified by the Japanese Society of Endoscopic Surgery (JSES) as experts in laparoscopic surgery performed RADG in all cases. To be board certified by the JSES as an expert laparoscopic surgeon, an applicant is required to perform more than 20 laparoscopic gastrectomies or alternative advanced laparoscopic surgeries within 3 years and to submit a nonedited video of one of the surgeries for a review by at least two board-qualified referees. The strict review process, which takes place once a year, allows only one-third of the applicants to be certified. Before introducing RADG at Shizuoka Cancer Center, the two surgeons completed a fixed training program for RADG as recommended by the JSES. The program consisted of e-learning, training sessions at an animal laboratory, and site visits to a specified high-volume center to observe actual RADG. In addition, surgeons with sufficient experience in RADG were invited as instructors in the initial two cases of RADG at our institution.

\section{Endpoints}

The primary endpoint in this study was the incidence of postoperative intraabdominal infectious complications, which included anastomotic leakage, pancreas-related infection, and intraabdominal abscess. Patients who developed Clavien-Dindo classification grade II or more complications by discharge were regarded as having complications [30, 31]. The secondary endpoints were overall survival (OS), relapse-free survival (RFS), RADG completion rate, and the incidence of all surgical morbidities.

Anastomotic leakage was diagnosed by radiologic examination using orally administered contrast media. Pancreas-related infection was defined as amylase-rich purulent discharge. Intraabdominal abscess was defined as an abscess not associated with anastomotic leakage or pancreas-related infection. The completion of RADG was defined as the proportion of patients without conversion from RADG to LADG or open distal gastrectomy (ODG).

Study design and statistical methods

In this phase II trial, the sample size was 18 cases, providing $70 \%$ power under the hypothesis of a primary endpoint with an expected value of $4 \%$ and a threshold 
value of $15 \%$, using one-sided testing at a $10 \%$ significance level. The expected value was decided according to the postoperative outcome of 265 patients who had undergone an ODG or LADG for early gastric cancer in the lower two-thirds of the stomach at the Shizuoka Cancer Center; the incidence of intraabdominal infectious complication among these patients was $4.5 \%$ [32]. All statistical analyses were conducted using $\mathrm{R}$ Statistics version 2.13.1.

\section{Results}

A total of 18 patients were recruited in this phase II study from May 2012 to November 2012. Table 1 summarizes the patient characteristics. The male-to-female ratio was 1.57 , median body mass index was $21.1 \mathrm{~kg} / \mathrm{m}^{2}$, and all patients had stage IA gastric cancer located within the lower two-thirds of the stomach. Undifferentiated histology was more frequently observed than differentiated histology.

Table 2 shows details of the surgical procedure. The median duration of the surgery was $311.5 \mathrm{~min}$; median docking, console, and anastomosis times were 22, 212.5, and $63 \mathrm{~min}$, respectively. Distal gastrectomy and pyloruspreserving gastrectomy were performed in nine patients

Table 1 Patient characteristics

\begin{tabular}{ll}
\hline Number of patients & 18 \\
Sex (cases) & 11 \\
Male & 7 \\
Female & \\
Age (years) & 65.5 \\
Median & $53-80$ \\
Range & \\
Body mass index $\left(\mathrm{kg} / \mathrm{m}^{2}\right)$ & 21.1 \\
Median & $16.2-25.8$ \\
Range & \\
Tumor location (cases) & 0 \\
Upper third & 11 \\
Middle third & 7 \\
Lower third & \\
Histological type (cases) & 6 \\
Differentiated & 12 \\
Undifferentiated & \\
Tumor size (cm) & \\
Median & \\
Range & \\
Clinical stage (cases) & \\
IA & \\
IB & \\
\hline
\end{tabular}

each, and all patients underwent D1 + lymph node dissection. No patient required conversion to laparoscopic or open surgery; thus, the RADG completion rate was $100 \%$. Median blood loss was $32.5 \mathrm{ml}$; blood transfusion was not required in any of the patients.

Postoperative clinical course is shown in Table 3. The median duration of postoperative hospital stay was 8 days. Incidence of intraabdominal infectious complication was $0 \%$ [0/18; $90 \%$ confidence interval (CI), 0-12.0\%]. The overall proportion of in-hospital adverse events was $22.2 \%$ (90\% CI, 8.0-43.9\%), with all rated as grade II, from which all patients recovered well with medical treatment only and no surgical interventions.

Table 2 Details of surgical procedures

\begin{tabular}{|c|c|}
\hline \multicolumn{2}{|l|}{ Operation time (min) } \\
\hline Median & 311.5 \\
\hline Range & $225-375$ \\
\hline \multicolumn{2}{|l|}{ Docking time (min) } \\
\hline Median & 22 \\
\hline Range & $11-41$ \\
\hline \multicolumn{2}{|l|}{ Console time (min) } \\
\hline Median & 212.5 \\
\hline Range & $161-291$ \\
\hline \multicolumn{2}{|l|}{ Anastomosis time (min) } \\
\hline Median & 63 \\
\hline Range & $41-111$ \\
\hline \multicolumn{2}{|l|}{ Blood loss (ml) } \\
\hline Median & 32.5 \\
\hline Range & $0-160$ \\
\hline \multicolumn{2}{|c|}{ Perioperative blood transfusion (cases) } \\
\hline Yes & 0 \\
\hline No & 18 \\
\hline \multicolumn{2}{|c|}{ Type of gastrectomy (cases) } \\
\hline PPG & 9 \\
\hline DG & 9 \\
\hline \multicolumn{2}{|l|}{ Reconstruction method } \\
\hline Roux-en-Y & 1 \\
\hline Billroth I & 8 \\
\hline Gastro-gastrostomy & 9 \\
\hline \multicolumn{2}{|c|}{ Extent of lymph node dissection (cases) } \\
\hline $\mathrm{D} 1+$ & 18 \\
\hline D2 & 0 \\
\hline \multicolumn{2}{|c|}{ Number of retrieved lymph nodes (cases) } \\
\hline Median & 40 \\
\hline Range & $26-89$ \\
\hline \multicolumn{2}{|c|}{ Completion of RADG (cases) } \\
\hline Yes & 0 \\
\hline No & 18 \\
\hline
\end{tabular}


Table 3 Postoperative clinical course

\begin{tabular}{ll}
\hline Postoperative hospital stay (days) & 8 \\
Median & $7-10$ \\
Range & \\
Postoperative morbidities (cases) & 0 \\
Intraabdominal infectious complications & 0 \\
Anastomotic leakage & 0 \\
Pancreas-related infection & 0 \\
Intraabdominal abscess & \\
Other complications & 2 \\
Wound infection & 1 \\
Delayed gastric emptying & 1 \\
Liver dysfunction & \\
\hline
\end{tabular}

\section{Discussion}

The present study showed RADG is feasible in terms of safety if experienced laparoscopic surgeons perform the surgery, with a zero incidence of intraabdominal infectious complications recorded (90\% CI, 0-12.0\%).

Before May 2012, we had performed five RADGs as an institute, and based on this experience, we assessed RADG as technically feasible. In addition, none of these five patients developed any postoperative complications. We therefore decided to more thoroughly assess the safety of RADG in the present prospective study.

Previous retrospective studies demonstrated that RADG was a feasible treatment for gastric cancer $[4,5,10,12,14$, 18 , 19]. Surgeons generally believed that much more meticulous surgery could be performed with the da Vinci Surgical System because of the three-dimensional surgical view provided and the flexibility of instrumentation. However, RADG required longer operation times [5, 14, 17-19, 21] and was more expensive than laparoscopic or open gastrectomy [14, 16, 21, 33]. In addition, the advantages of RADG compared to conventional procedures were not clear from these previous studies, and no prospective study investigating the safety of RADG was reported.

The incidence of postoperative intraabdominal infectious complication in the present study was $0 \%(90 \% \mathrm{CI}$, $0-12.2 \%$ ) with a $22.2 \%$ overall proportion of in-hospital adverse events (90\% CI, 8.0-43.9\%) in this study. A similar complication rate $(0-47.3 \%)$ has been reported in previous retrospective studies, although none had focused on the incidence of intraabdominal infectious complication $[4,5,8-10,12,17,18,22,33]$. With the three-dimensional magnified view available with RADG, surgeons were able to recognize anatomical structures much more precisely than with the standard two-dimensional view. In addition, the flexibility of instruments used helped surgeons perform meticulous surgery. We propose that these advantages of RADG resulted in the low complication rate.
Other possible reasons for the low complication rate recorded in this study were involving only experienced laparoscopic surgeons to perform the procedures and the relatively lower BMI of the patients compared with that reported in Western series. High BMI is a possible risk factor for postoperative complications after open and laparoscopic gastrectomy, although this association remains controversial [34-38]. The present study included only one overweight patient (BMI, 25.8). The feasibility of RADG in overweight or obese patients is still unclear and must be clarified in a future trial.

RADG procedures required longer surgical times than LADG. Indeed, there was a difference of $86.5 \mathrm{~min}$ in our institute between RADG and LADG [31]. We considered that the meticulousness of the procedure was inversely proportional to operation time to some degree. With the magnified and three-dimensional magnified view and instrument flexibility, surgeons were able to perform much more meticulous surgery at the expense of increased operation time.

There were other possible reasons for the longer operation times. First, RADG was performed during our learning curve period whereas LADG was not. Second, we did not use ultrasonic shears provided for RADG because such usage is not allowed in Japan with the da Vinci Surgical System. Thus, if we achieve our learning curve with RADG and the usage of ultrasonic devices is permitted in the future, we will be able to reduce the operation time.

We believe that the advantages of the da Vinci Surgical System would be enhanced when we use it for more complicated surgery such as gastrectomy with extended (D2) lymph node dissection or mediastinal lymph node dissection. During extended lymph node dissection, we were able to recognized layers precisely as well as small vessels because of the three-dimensional magnified view. In addition, the flexibility of instruments and tremor suppression enabled us to do each procedure meticulously, resulting in high-quality lymph node dissection. Similar advantages would be obtained when we perform lower mediastinal lymph node dissection for adenocarcinoma of esophagogastric junction in which the surgical field is narrow and linear instruments used in laparoscopic surgery frequently interfere. Thus, our next step is to indicate the da Vinci Surgical System for these complicated surgeries.

In the present study, early surgical outcomes of RADG were not compared with conventional open or laparoscopic surgery; thus, it is still unclear if RADG is superior to conventional surgeries in this regard. Although previous retrospective studies compared surgical outcomes of RADG with LADG or ODG, there is no prospective randomized trial comparing RADG and other procedures [5, $17,18,20,22]$. In addition, survival outcomes of RADG 
remain unclear. Future trials are needed to clarify the superiority of RADG over other procedures, including both short- and long-term outcomes, before it can be accepted as a standard treatment for gastric cancer.

The present study had limitations including the relatively small sample size. The Japanese national insurance system does not reimburse patients for RADG; thus, either the patient or the hospital has to pay the entire admission fee in addition to the surgical fee (around USD \$20,000). It was therefore challenging to recruit sufficient patient numbers even for a small phase II trial, and so long as this situation persists, the cost of such surgeries will be an issue and the forthcoming surgeries will be paid by the hospital or the patient in our hospital. We consider that the future practical use of RADG in Japan as an advanced medical technology will require a well-planned prospective trial involving sufficient patient numbers to provide important information about issues such as reimbursement. However, we also believe that accumulated evidence from smaller prospective studies such as ours will help future, largerscale trials for RADG.

In conclusion, this early phase II study suggested that RADG might be a safe and feasible procedure for stage IA gastric cancer, providing experienced surgeons perform the surgery. This conclusion should be clarified in subsequent late phase II studies with a larger sample size.

\section{References}

1. Kim HH, Hyung WJ, Cho GS, Kim MC, Han SU, Kim W, Ryu SW, et al. Morbidity and mortality of laparoscopic gastrectomy versus open gastrectomy for gastric cancer: an interim report. A phase III multicenter, prospective, randomized trial (KLASS Trial). Ann Surg. 2010;251(3):417-20.

2. Katai H, Sasako M, Fukuda H, Nakamura K, Hiki N, Saka M, et al. Safety and feasibility of laparoscopy-assisted distal gastrectomy with supra-pancreatic nodal dissection for clinical stage I gastric cancer: a multicenter phase II trial (JCOG 0703). Gastric Cancer. 2010;13(4):238-44.

3. Nakamura K, Katai H, Mizusawa J, Yoshikawa T, Ando M, Terashima M, et al. A phase III study of laparoscopy-assisted versus open distal gastrectomy with nodal dissection for clinical stage IA/IB gastric cancer (JCOG0912). Jpn J Clin Oncol. 2013;43(3):324-7.

4. Song J, Oh SJ, Kang WH, Hyung WJ, Choi SH, Noh SH. Robotassisted gastrectomy with lymph node dissection for gastric cancer: lessons learned from an initial 100 consecutive procedures. Ann Surg. 2009;249(6):927-32.

5. Song J, Kang WH, Oh SJ, Hyung WJ, Choi SH, Noh SH. Role of robotic gastrectomy using da Vinci system compared with laparoscopic gastrectomy: initial experience of 20 consecutive cases. Surg Endosc. 2009;23(6):1204-11.

6. Hashizume M, Sugimachi K. Robot-assisted gastric surgery. Surg Clin N Am. 2003;83(6):1429-44.

7. Kakeji Y, Konishi K, Ieiri S, Yasunaga T, Nakamoto M, Tanoue $\mathrm{K}$, et al. Robotic laparoscopic distal gastrectomy: a comparison of the da Vinci and Zeus systems. Int J Med Robot. 2006;2(4): 299-304.

8. Isogaki J, Haruta S, Man IM, Suda K, Kawamura Y, Yoshimura F, et al. Robot-assisted surgery for gastric cancer: experience at our institute. Pathobiol J Immunopathol Mol Cell Biol. 2011;78(6):328-33.

9. Park SS, Kim MC, Park MS, Hyung WJ Rapid adaptation of robotic gastrectomy for gastric cancer by experienced laparoscopic surgeons. Surg Endosc. 2012;26(1):60-7.

10. Lee HH, Hur H, Jung H, Jeon HM, Park CH, Song KY. Robotassisted distal gastrectomy for gastric cancer: initial experience. Am J Surg. 2011;201(6):841-5.

11. Heemskerk J, van Gemert WG, de Vries J, Greve J, Bouvy ND. Learning curves of robot-assisted laparoscopic surgery compared with conventional laparoscopic surgery: an experimental study evaluating skill acquisition of robot-assisted laparoscopic tasks compared with conventional laparoscopic tasks in inexperienced users. Surg Laparosc Endosc Percutan Tech. 2007;17:171-4.

12. D’Annibale A, Pende V, Pernazza G, Monsellato I, Mazzocchi P, Lucandri G, et al. Full robotic gastrectomy with extended (D2) lymphadenectomy for gastric cancer: surgical technique and preliminary results. J Surg Res. 2011;166(2):e113-20.

13. Pugliese R, Maggioni D, Sansonna F, Costanzi A, Ferrari GC, Di Lernia S, et al. Subtotal gastrectomy with D2 dissection by minimally invasive surgery for distal adenocarcinoma of the stomach: results and 5-year survival. Surg Endosc. 2010;24(10):2594-602.

14. Patriti A, Ceccarelli G, Bellochi R, Bartoli A, Spaziani A, Di Zitti $\mathrm{L}$, et al. Robot-assisted laparoscopic total and partial gastric resection with D2 lymph node dissection for adenocarcinoma. Surg Endosc. 2008;22(12):2753-60.

15. Pugliese R, Maggioni D, Sansonna F, Ferrari GC, Forgione A, Costanzi A, et al. Outcomes and survival after laparoscopic gastrectomy for adenocarcinoma. Analysis on 65 patients operated on by conventional or robot-assisted minimal access procedures. Eur J Surg Oncol. 2009;35(3):281-8.

16. Anderson C, Ellenhorn J, Hellan M, Pigazzi A. Pilot series of robotassisted laparoscopic subtotal gastrectomy with extended lymphadenectomy for gastric cancer. Surg Endosc. 2007;21(9):1662-6.

17. Kim MC, Heo GU, Jung GJ. Robotic gastrectomy for gastric cancer: surgical techniques and clinical merits. Surg Endosc. 2010;24(3):610-5.

18. Woo Y, Hyung WJ, Pak KH, Inaba K, Obama K, Choi SH et al. Robotic gastrectomy as an oncologically sound alternative to laparoscopic resections for the treatment of early-stage gastric cancers. Arch Surg. 2011;146(9):1086-92.

19. Caruso S, Patriti A, Marrelli D, Ceccarelli G, Ceribelli C, Roviello $\mathrm{F}$, et al. Open vs. robot-assisted laparoscopic gastric resection with D2 lymph node dissection for adenocarcinoma: a case-control study. Int J Med Robot. 2011;7(4):452-8.

20. Yoon HM, Kim YW, Lee JH, Ryu KW, Eom BW, Park JY, et al. Robot-assisted total gastrectomy is comparable with laparoscopically assisted total gastrectomy for early gastric cancer. Surg Endosc. 2012;26(5):1377-81.

21. Park JY, Jo MJ, Nam BH, Kim Y, Eom BW, Yoon HM, et al. Surgical stress after robot-assisted distal gastrectomy and its economic implications. Br J Surg. 2012;99(11):1554-61.

22. Hyun MH, Lee CH, Kwon YJ, Cho SI, Jang YJ, Kim DH et al. Robot versus laparoscopic gastrectomy for cancer by an experienced surgeon: comparisons of surgery, complications, and surgical stress. Ann Surg Oncol. 2013;20(4):1258-65.

23. Sobin L, Gospodarowicz M, Wittekind C. TNM classification of malignant tumors. 7th ed. New York: Wiley-Blackwell; 2009.

24. Japanese Gastric Cancer Association. Japanese gastric cancer treatment guidelines 2010 (ver. 3). Gastric Cancer. 2011;14(2): $113-23$. 
25. Sano T, Aiko T. New Japanese classifications and treatment guidelines for gastric cancer: revision concepts and major revised points. Gastric Cancer. 2011;14(2):97-100.

26. Hiki N, Fukunaga T, Yamaguchi T, Nunobe S, Tokunaga M, Ohyama S, et al. The benefits of standardizing the operative procedure for the assistant in laparoscopy-assisted gastrectomy for gastric cancer. Langenbecks Arch Surg. 2008;393(6):963-71.

27. Fukunaga T, Hiki N, Tokunaga M, Nohara K, Akashi Y, Katayama $\mathrm{H}$, et al. Left-sided approach for supra-pancreatic lymph node dissection in laparoscopy-assisted distal gastrectomy without duodenal transection. Gastric Cancer. 2009;12(2):106-12.

28. Nunobe S, Hiki N, Fukunaga T, Tokunaga M, Ohyama S, Seto Y, et al. Laparoscopy-assisted pylorus-preserving gastrectomy: preservation of vagus nerve and infrapyloric blood flow induces less stasis. World J Surg. 2007;31(12):2335-40.

29. Tokunaga M, Hiki N, Fukunaga T, Ohyama S, Nunobe S, Yamada $\mathrm{K}$, et al. Is preservation of the celiac branch of the vagal nerve effective in preventing stasis following pylorus-preserving gastrectomy? Hepatogastroenterology. 2011;58(107108):1046-50.

30. Dindo D, Demartines N, Clavien PA. Classification of surgical complications: a new proposal with evaluation in a cohort of 6336 patients and results of a survey. Ann Surg. 2004;240(2): 205-13.

31. Clavien PA, Barkun J, de Oliveira ML, Vauthey JN, Dindo D, Schulick RD, et al. The Clavien-Dindo classification of surgical complications: five-year experience. Ann Surg. 2009;250(2): 187-96.

32. Tokunaga M, Kondo J, Tanizawa Y, Bando E, Kawamura T, Terashima M. Postoperative intra-abdominal complications assessed by the Clavien-Dindo classification following open and laparoscopy-assisted distal gastrectomy for early gastric cancer. J Gastrointest Surg. 2012.

33. Clark J, Sodergren MH, Purkayastha S, Mayer EK, James D, Athanasiou $\mathrm{T}$, et al. The role of robotic assisted laparoscopy for esophagogastric oncological resection; an appraisal of the literature. Dis Esophagus. 2011;24(4):240-50.

34. Tokunaga M, Hiki N, Fukunaga T, Ogura T, Miyata S, Yamaguchi T. Effect of individual fat areas on early surgical outcomes after open gastrectomy for gastric cancer. Br J Surg. 2009;96(5): 496-500.

35. Noshiro H, Shimizu S, Nagai E, Ohuchida K, Tanaka M. Laparoscopy-assisted distal gastrectomy for early gastric cancer: is it beneficial for patients of heavier weight? Ann Surg. 2003;238(5): $680-5$.

36. Yamada H, Kojima K, Inokuchi M, Kawano T, Sugihara K. Effect of obesity on technical feasibility and postoperative outcomes of laparoscopy-assisted distal gastrectomy: comparison with open distal gastrectomy. J Gastrointest Surg. 2008;12(6): 997-1004.

37. Makino $\mathrm{H}$, Kunisaki $\mathrm{C}$, Izumisawa $\mathrm{Y}$, Tokuhisa $\mathrm{M}$, Oshima $\mathrm{T}$, Nagano Y, et al. Effect of obesity on laparoscopy-assisted distal gastrectomy compared with open distal gastrectomy for gastric cancer. J Surg Oncol. 2010;102(2):141-7.

38. Hiki N, Fukunaga T, Yamaguchi T, Ogura T, Miyata S, Tokunaga $\mathrm{M}$, et al. Increased fat content and body shape have little effect on the accuracy of lymph node retrieval and blood loss in laparoscopic distal gastrectomy for gastric cancer. J Gastrointest Surg. 2009;13(4):626-33. 